\title{
Lenvatinib and Subsequent Therapy for Radioactive Iodine-Refractory Differentiated Thyroid Cancer: A Real-World Study of Clinical Effectiveness in the United States
}

\author{
Jonathan K. Kish (D) - Debanjana Chatterjee - Yin Wan • \\ Hsing-Ting Yu • Djibril Liassou • Bruce A. Feinberg
}

Received: March 10, 2020 / Published online: May 7, 2020

(C) The Author(s) 2020

\begin{abstract}
Introduction: Lenvatinib has become the most commonly prescribed first-line $(1 \mathrm{~L})$ agent for the treatment of radioactive iodine-refractory differentiated thyroid cancer (RAI-r DTC) since its approval in 2015. With no real-world studies describing clinical outcomes of $1 \mathrm{~L}$ lenvatinib and subsequent therapy, the current study aimed to assess treatment sequencing and related clinical outcomes in patients treated with $1 \mathrm{~L}$ lenvatinib in the USA

Methods: We conducted a multisite, retrospective chart review of US patients with a diagnosis of RAI-r DTC who had initiated 1L therapy with lenvatinib from January 1, 2016 through May 31, 2017 with follow-up through October 17, 2018. Physicians completed electronic case
\end{abstract}

Digital Features To view digital features for this article go to https://doi.org/10.6084/m9.figshare.12179448.

J. K. Kish $(\bowtie)$ · H.-T. Yu · D. Liassou · B. A. Feinberg Real-World Evidence and Insights, Cardinal Health Specialty Solutions, Dublin, OH, USA

e-mail: jonathan.kish@cardinalhealth.com

D. Chatterjee

US Health Economics and Outcomes Research \& Real-World Evidence, Formerly of Eisai Inc.,

Woodcliff Lake, NJ, USA

Y. Wan

US Health Economics and Outcomes Research \& Real-World Evidence, Eisai Inc., Woodcliff Lake, NJ, USA report forms for two patient cohorts: patients still receiving $1 \mathrm{~L}$ lenvatinib (cohort 1 ) and those who had initiated second-line (2L) therapy prior to data cutoff (cohort 2). Real-world objective response rate (ORR) was assessed for both cohorts. Progression-free survival (PFS) and overall survival (OS) were assessed for cohort 2 . Results: A total of 252 patients met the study criteria with 71 in cohort 1 and 181 in cohort 2 . Patients were predominantly female, had papillary DTC, and had lung metastases. The ORR was $64.8 \%$ for cohort 1 and $53.6 \%$ for cohort 2 . In cohort 2 , median PFS from 1L lenvatinib initiation was 14.0 months (95\% CI 12.7-15.0). Second-line treatments included sorafenib (49.7\%), cabozantinib (19.3\%), and other targeted/chemotherapy/immuno-oncology agents. The ORR in $2 \mathrm{~L}$ therapy was $15.5 \%$. For cohort 2, the 12-, 18-, and 24-month OS from initiation of $1 \mathrm{~L}$ lenvatinib was $92.8 \%, 81.5 \%$, and $66.9 \%$, respectively.

Conclusions: In this first real-world examination of clinical effectiveness of $1 \mathrm{~L}$ lenvatinib and subsequent therapy among patients in the US, the results demonstrated that treatment with $1 \mathrm{~L}$ lenvatinib followed by another $2 \mathrm{~L}$ therapy may deliver a clinical benefit, thus allowing a number of potential 2L options following $1 \mathrm{~L}$ lenvatinib for patients with RAI-r DTC.

Keywords: Effectiveness; Lenvatinib; Progression-free survival; Radioiodine refractory; Realworld; Response; Thyroid 


\section{Key Summary Points}

Why carry out this study?

Lenvatinib is the most commonly prescribed first-line treatment for radioactive iodine-refractory differentiated thyroid cancer (RAI-r DTC).

This was the first and largest study to explore real-world clinical outcomes of first-line lenvatinib in addition to treatment patterns and outcomes in second-line post-lenvatinib among patients with RAI-r DTC in the USA.

\section{What was learned from the study?}

Real-world clinical outcomes of first-line lenvatinib therapy were similar to that observed in the randomized controlled trial.

Patients derived a clinical benefit from multiple different agents and classes of agents in second-line after first-line lenvatinib.

\section{INTRODUCTION}

Differentiated thyroid cancer (DTC) comprises over $90 \%$ of the estimated 52,070 cases of thyroid cancer that will be diagnosed in the USA in 2019 [1, 2] With multi-modality treatment, including thyroidectomy, the 5-year survival rate for stage I-III DTC is over $90 \%$ and the majority of patients are cured of disease [3]. Standard treatment for patients with high-risk disease (stage III or IV) is radioactive iodine (RAI) therapy, which has been shown to decrease disease progression and mortality [4]. However, for the roughly $30 \%$ of patients who develop recurrence or distant metastases and who become RAI refractory (RAI-r), disease prognosis is poor: the 10-year survival rate is approximately $10 \%$ and the mean life expectancy is $3-5$ years [4-10]

For patients with RAI-r DTC, systemic chemotherapy has limited efficacy and high toxicity and was the only option for treatment prior to 2012. In 2012, the first multikinase inhibitor (MKI), sorafenib was approved for RAIr DTC. Subsequently in 2015, lenvatinib was approved on the basis of improved objective response rates (ORR) and prolonged progression-free survival (PFS) compared to placebo $[11,12]$. Lenvatinib, an MKI (inhibitor of VEGF, FGFR, PDGFR $\alpha$, RET, and KIT), was approved on the basis of the SELECT trial (lenvatinib versus placebo) [12]. In both patients who were treatment naïve and those with prior VEGF-based therapies, lenvatinib significantly improved ORR $(64.8 \%$ versus $1.5 \%, p<0.001)$ and PFS (median 18.3 months versus 3.6 months) [13]. While the median overall survival (OS) was not reached, the risk of progression or death was reduced in the lenvatinib arm by $79 \%$ $(p<0.0001)$.

A recent administrative claims study from the USA demonstrated that lenvatinib is the most commonly prescribed first-line (1L) agent for the treatment of RAI-r DTC [14]. However, no real-world clinical outcomes for patients who receive $1 \mathrm{~L}$ lenvatinib in the USA have been reported previously. Moreover, the last 5 years have also seen a significant expansion of therapeutic agents for $2 \mathrm{~L}$ and beyond with the approvals of pazopanib, sunitinib, vandetinib, and cabozantinib. These drugs were evaluated in clinical trials in patients previously treated with sorafenib. No outcomes data exists for these therapies post lenvatinib even from clinical trials.

The main objective of the current study was to assess the real-world clinical effectiveness for patients with RAI-r DTC treated with 1L lenvatinib. In addition, treatment sequence in second-line (2L) and the clinical outcomes of $2 \mathrm{~L}$ therapy following $1 \mathrm{~L}$ lenvatinib were assessed as a secondary objective.

\section{METHODS}

\section{Study Design}

This study was a real-world, retrospective, multisite patient chart review. Physicians actively caring for patients with RAI-r DTC who were 
part of the Cardinal Health Oncology Provider Extended Network (OPEN) were invited to participate. OPEN is an informal group of over 7000 oncologists and hematologists practicing in community oncology clinics across the USA. Those providers who had treated at least one patient with RAI-r DTC with lenvatinib were asked to select up to 10 patients whom they personally cared for at their practice meeting the study selection criteria including confirmed diagnosis of RAI-r DTC, initiated 1L lenvatinib between January 1, 2016 and May 31, 2017, 18 years of age or older at initiation of lenvatinib, and disease response assessment to $1 \mathrm{~L}$ lenvatinib therapy completed via radiographic imaging. In addition, we purposefully split the sample selection into two cohorts: cohort 1 consisted of patients who were still receiving $1 \mathrm{~L}$ lenvatinib at the time of data cutoff (Fig. 1a) and cohort 2 consisted of patients who had received $1 \mathrm{~L}$ lenvatinib, but during the study follow-up period had discontinued 1L lenvatinib as a result of disease progression, toxicity, or patient choice and initiated 2L therapy (Fig. 1b). Patients who were participants in any lenvatinib clinical trial or those with synchronous anaplastic histology were excluded.

Only the physician abstractors had access to the selected patients' electronic health record data. The physician completed an electronic case report form (eCRF) abstracting relevant demographics, treatments for RAI-r DTC, and clinical markers of prognosis at diagnosis (e.g., stage, performance status, comorbidities), genetic abnormalities (e.g., BRAF, RAS, RET), drug therapies received (including start and stop dates and reasons for discontinuation), disease response, dates of disease progression, and date of death (if deceased) from each patient chart. Data collection occurred between September 27, 2018 and October 17, 2018 (Fig. 1). Prior to data collection the research protocol and eCRF were reviewed by the Western Institutional Review Board (IRB) (Study Number: 1187046). The IRB found that this research meets the requirements for a waiver of consent under 45 CFR 46.116(d).

To ensure data reliability, each submitted eCRF was reviewed by clinical staff for clinical outliers and a separate $10 \%$ random sample of
eCRFs was selected for data verification with the provider. Data verification was accomplished by asking the provider to re-enter three data points for the selected eCRF. Patients for whom the data were not able to be verified were excluded from the analysis.

Response data were required to be verified by radiographic imaging and imaging results available (tumor lesion measurements) in order to perform a retrospective calculation of disease response based on RECIST v1.1 guidelines [15]. The physician data abstractors were asked to abstract the sites and measurements for all lesions presented and identified at baseline (prior to initiation of $1 \mathrm{~L}$ and $2 \mathrm{~L}$ ) and at the time of best response to therapy in each line of therapy. Physicians were asked to consult all available imaging reports or the images themselves to identify the lesions and their measurements. Using these lesion measurements (not the radiographic images themselves), the research team calculated disease response based on the RECIST algorithm. In instances where the provider could not provide the measurements at baseline and then of the lesion at best response, the response was assigned as not evaluable.

\section{Data Analysis}

Analyses were conducted separately for cohort 1 and cohort 2 to minimize confounding in our estimates of $1 \mathrm{~L}$ lenvatinib effectiveness. Descriptive measures including counts and frequencies for dichotomous and categorical variables and measures of centrality (mean, median) and spread (min, max, standard deviation [SD], interquartile range, as appropriate) for continuous variables were used to summarize patient demographics, clinical characteristics, treatment regimens received in $2 \mathrm{~L}$ (for cohort 2), and the ORR in a real-world setting. The ORR in $1 \mathrm{~L}$ and $2 \mathrm{~L}$ (for cohort 2 only) was calculated as the proportion of patients with a complete response (CR) or partial response (PR) with regard to all patients. PFS and OS were calculated for cohort 2 patients only (as all cohort 1 patients were still receiving 1L lenvatinib therapy at the time of data cutoff and no 


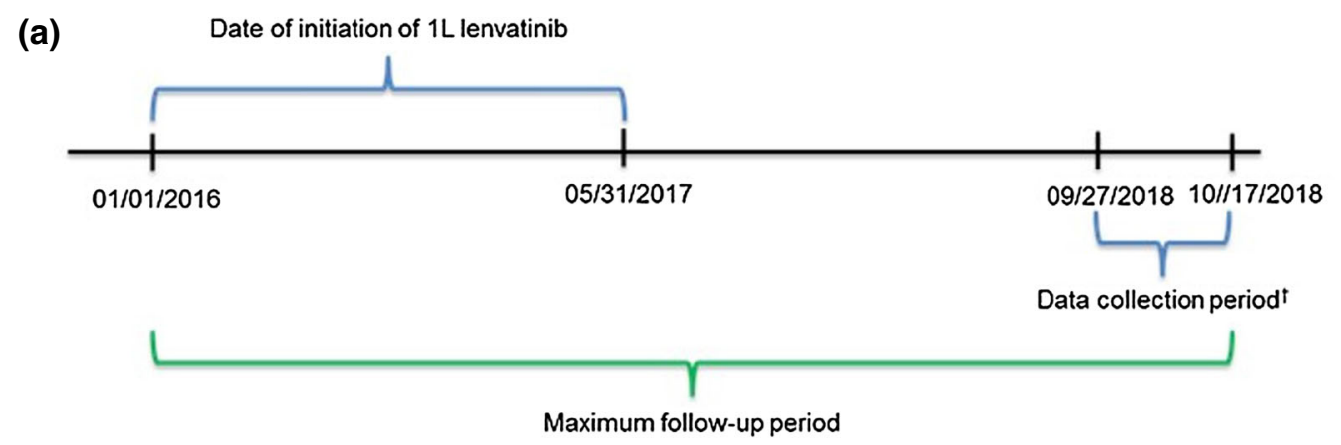

(b) Date of initiation of any $2 L$ therapy (anytime after discontinuation of $1 L$ lenvatinib

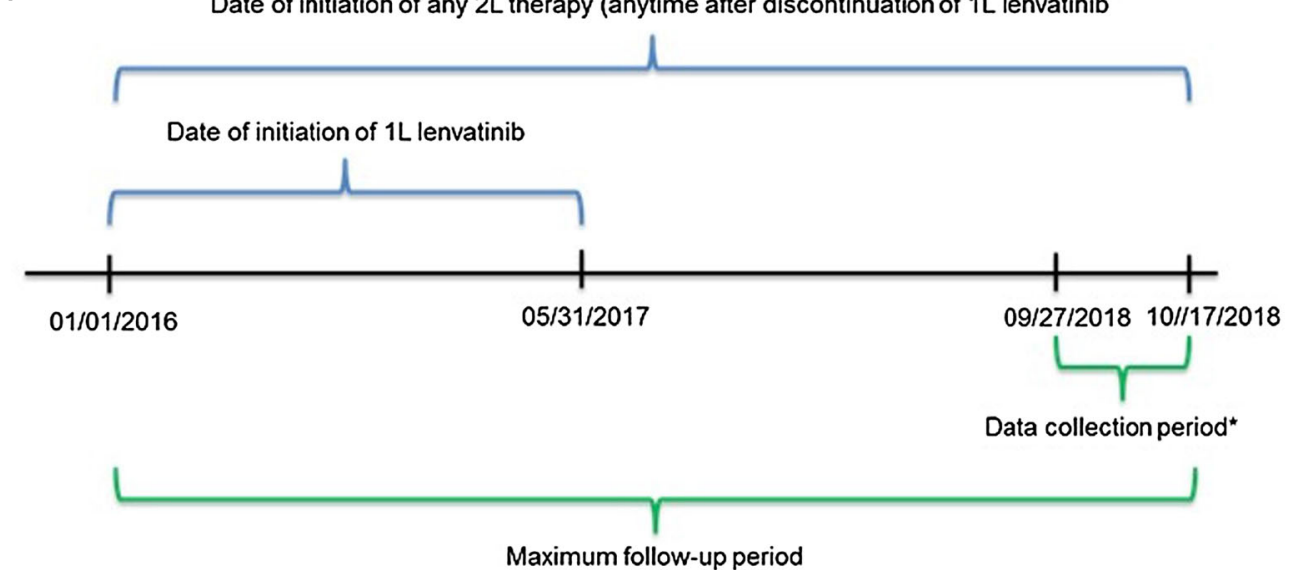

Fig. 1 Study period: a cohort $1, \mathbf{b}$ cohort $2 .{ }^{\dagger}$ Patients were still receiving $1 \mathrm{~L}$ lenvatinib at the time of data cutoff. ${ }^{*}$ Patients may still have been receiving $2 \mathrm{~L}$ treatment at the time of data cutoff. $1 \mathrm{~L}$ first-line, $2 \mathrm{~L}$ second-line

events of death or progression were observed at data cutoff date) using the Kaplan-Meier (KM) method. PFS was calculated separately in cohort 2 for $1 \mathrm{~L}$ lenvatinib and $2 \mathrm{~L}$ (from the date of initiation of the line of therapy). For PFS calculation, an event was any discontinuation of therapy due to disease progression or death; patients who discontinued therapy as a result of any other reason were censored on the date of discontinuation. Patients still receiving therapy at the time of data cutoff were censored on their last visit with the provider. The median PFS and PFS at $6,12,18$, and 24 months (and 95\% confidence intervals [CIs]) post initiation of a line of therapy are reported. Survival rates are reported at 6,12 , and 18 months post initiation of $1 \mathrm{~L}$ lenvatinib for cohort 2 . All analyses were performed with SAS v9.4.

\section{RESULTS}

\section{Study Sample}

Overall, 261 eCRFs were submitted, of which 9 (3.4\%) were excluded during data verification. Providers reported an average of 8.4 eligible patients and completed data abstraction for $73.2 \%$ of those eligible. Of the 252 patients who met the study criteria, 71 met the cohort 1 criteria and 181 met the cohort 2 criteria. The 252 total patients came from 31 unique community practices in the USA, of which $35.5 \%$ of practices were in the South, $29.0 \%$ in the Northeast, $22.6 \%$ in the West, and $12.9 \%$ in the Midwest.

\section{Characteristics and Outcomes for Cohort 1}

The majority of patients in cohort 1 were female $(53.5 \%)$, white race $(76.1 \%)$, and were on 
average 58.3 years of age at initiation of lenvatinib (SD $=10.7$ years) (Table 1$)$. The majority of patients were either American Joint Committee on Cancer (AJCC) stage III $(26.8 \%)$ or stage IV (49.4\%). The mean cumulative dose of iodine131 received prior to initiation of lenvatinib was $276.0 \mathrm{mCi}$ (standard deviation = 233.4). Mean time from initial diagnosis to RAI-r disease was 34.6 months $(\mathrm{SD}=39.1)$ and from initial diagnosis to initiation of $1 \mathrm{~L}$ lenvatinib was 35.7 months $(\mathrm{SD}=40.2)$. The most frequent histological type was papillary DTC (62.0\%). Most patients had an Eastern Cooperative Oncology Group performance status (ECOG-PS) $0 / 1$ at initiation of $1 \mathrm{~L}$ therapy $(87.3 \%)$ and had metastases to the lung (71.8\%). Genetic mutations of $B R A F$ were assessed in up to $46.5 \%$ of patients, of which $27.3 \%$ were positive. The rate of testing and prevalence (in all patients) of other mutations were, respectively, $39.4 \%$ and $17.9 \%$ for $R A S ; 46.5 \%$ and $18.2 \%$ for $R E T ; 39.4 \%$ and $10.7 \%$ for PI3K; and $35.2 \%$ and $0.0 \%$ for PTEN. Approximately $48.3 \%$ of mutational testing occurred prior to the development of RAI-r disease and $51.7 \%$ post RAI-r. Mean follow-up from initiation of $1 \mathrm{~L}$ lenvatinib was 17.8 months $(\mathrm{SD}=5.8)$ and $90.1 \%$ of patients $(n=64)$ initiated lenvatinib at $24 \mathrm{mg}$ once daily. Lenvatinib dose reductions (last dose administered less than starting dose) were reported in 12 (16.9\%) patients with 10/12 due to toxicity and 10/12 reductions were from starting dose of $24 \mathrm{mg}$, one with the starting dose of $20 \mathrm{mg}$, and one with the starting dose of $14 \mathrm{mg}$. Sixty percent of patients with a dose reduction after starting at $24 \mathrm{mg}$ reduced to $20 \mathrm{mg}$.

The ORR was $64.8 \%$ (Table 2) including six CRs (8.5\%) and 40 PRs (56.3\%); 10 patients experienced SD (14.1\%) and 10 patients experienced PD (14.1\%). Per the cohort definition, no patients in cohort 1 had discontinued $1 \mathrm{~L}$ lenvatinib at the time of data cutoff and thus PFS/OS data were not available for this cohort.

\section{Characteristics and Outcomes for Cohort 2}

Demographic and clinical characteristics of cohort 2 are presented in Table 1 . The majority of patients were female $(57.5 \%)$, white race $(76.2 \%)$, diagnosed with papillary DTC $(56.4 \%)$, and diagnosed at either stage III $(24.9 \%)$ or stage IV (39.2\%). The mean cumulative dose of iodine-131 received prior to initiation of lenvatinib was $216.8 \mathrm{mCi}$ (standard deviation $=230.7$ ). Mean time from initial diagnosis to RAI-r disease was 29.8 months ( $\mathrm{SD}=26.7$ ) and from initial diagnosis to initiation of $1 \mathrm{~L}$ lenvatinib was 32.0 months $(S D=28.7)$. The majority of patients initiated lenvatinib at $24 \mathrm{mg}$ once daily (93.4\%). Although rates of genetic testing were very similar between cohort 1 and cohort 2 , a higher proportion of cohort 2 patients tested positive for BRAF $(48.1 \%$ versus $27.3 \%), \quad R A S \quad(50.0 \%$ versus $17.9 \%)$, RET (41.8\% versus $18.2 \%)$, and PTEN (2.0\% versus $0.0 \%$ ) while a lower proportion were positive for PI3K (9.1\% versus $10.7 \%)$. Approximately $48.2 \%$ of mutational testing occurred prior to the development of RAI-r disease and $51.8 \%$ post RAI-r. Mean follow-up from initiation of 1L lenvatinib in cohort 2 was 20.5 months $(S D=6.0)$. Dose reductions were reported in $11.1 \%$ of patients $(n=20), 19 / 20$ were due to toxicity, and 17/20 reductions were from starting dose of $24 \mathrm{mg}$, one from starting dose of $20 \mathrm{mg}$, and two from starting dose of $16 \mathrm{mg}$. Fifty percent of patients with a dose reduction after starting at $24 \mathrm{mg}$ reduced to $20 \mathrm{mg}$.

As defined by the study inclusion criteria, all patients in cohort 2 had discontinued 1L lenvatinib and initiated 2L therapy. Of the 181 patients, $162(89.5 \%)$ discontinued 1L lenvatinib as a result of disease progression, $16(9.8 \%)$ as a result of patient request, and $3(1.7 \%)$ as a result of toxicity. The ORR to $1 \mathrm{~L}$ lenvatinib in cohort 2 was $53.6 \%$ (Table 2). Median PFS from initiation of $1 \mathrm{~L}$ lenvatinib in cohort 2 was 14.0 months (95\% CI $12.7-15.0)$ (Table 3). Median time to progression was 13.7 months among patients who discontinued 1L lenvatinib as a result of disease progression $(n=162)$. At 6 months post 1L lenvatinib initiation, $86.0 \%$ (95\% CI $80.9-91.1 \%$ ) of patients were progression-free, at 12 months $60.3 \% \quad(95 \%$ CI $53.0-67.6 \%)$, at 18 months $21.2 \%$ (95\% CI $14.8-27.5 \%)$, and at 24 months $2.1 \%(95 \% \mathrm{CI}$ $0.0-4.5 \%)$. 
Table 1 Patient demographics, clinical characteristics, and lenvatinib dosing

\begin{tabular}{|c|c|c|c|c|}
\hline & \multicolumn{2}{|c|}{ Cohort 1} & \multicolumn{2}{|l|}{ Cohort 2} \\
\hline & $n=71$ & & $n=181$ & \\
\hline \multicolumn{5}{|l|}{ Gender $(n, \%)$} \\
\hline Male & 33 & $46.5 \%$ & 77 & $42.5 \%$ \\
\hline Female & 38 & $53.5 \%$ & 104 & $57.5 \%$ \\
\hline \multicolumn{5}{|l|}{ Patient race $(n, \%)$} \\
\hline White & 54 & $76.1 \%$ & 138 & $76.2 \%$ \\
\hline Asian & 4 & $5.6 \%$ & 9 & $5.0 \%$ \\
\hline Black/African American & 8 & $11.3 \%$ & 26 & $14.4 \%$ \\
\hline Native Hawaiian or Other Pacific Islander & 1 & $1.4 \%$ & 0 & $0.0 \%$ \\
\hline American Indian or Alaska Native & 1 & $1.4 \%$ & 0 & $0.0 \%$ \\
\hline Other & 3 & $4.2 \%$ & 8 & $4.4 \%$ \\
\hline \multicolumn{5}{|l|}{ Histological subtype of DTC $(n, \%)$} \\
\hline Follicular & 27 & $38.0 \%$ & 74 & $40.9 \%$ \\
\hline Papillary & 44 & $62.0 \%$ & 102 & $56.4 \%$ \\
\hline Hürthle cell & 0 & $0.0 \%$ & 5 & $2.8 \%$ \\
\hline \multicolumn{5}{|l|}{ Tumor characteristics at initial diagnosis $(n, \%)$} \\
\hline Extrathyroid extension & 21 & $29.6 \%$ & 42 & $23.2 \%$ \\
\hline Multifocality & 24 & $33.8 \%$ & 42 & $23.2 \%$ \\
\hline Vascular invasion & 32 & $45.1 \%$ & 67 & $37.0 \%$ \\
\hline \multicolumn{5}{|l|}{ AJCC stage at initial diagnosis } \\
\hline $\mathrm{I}$ & 2 & $2.8 \%$ & 2 & $1.1 \%$ \\
\hline II & 12 & $16.9 \%$ & 26 & $14.4 \%$ \\
\hline III & 19 & $26.8 \%$ & 45 & $24.9 \%$ \\
\hline IVA & 11 & $15.5 \%$ & 20 & $11.0 \%$ \\
\hline IVB & 6 & $8.5 \%$ & 5 & $2.8 \%$ \\
\hline IVC & 18 & $25.4 \%$ & 46 & $25.4 \%$ \\
\hline Unknown & 3 & $4.2 \%$ & 37 & $20.4 \%$ \\
\hline \multicolumn{5}{|c|}{ Genetic mutations (\% tested, $\%$ mutated of those tested) } \\
\hline$B R A F$ & $46.5 \%$ & $27.3 \%$ & $43.1 \%$ & $48.1 \%$ \\
\hline$R A S$ & $39.4 \%$ & $17.9 \%$ & $39.2 \%$ & $50.0 \%$ \\
\hline RET & $46.5 \%$ & $18.2 \%$ & $44.8 \%$ & $41.8 \%$ \\
\hline$P I 3 K$ & $39.4 \%$ & $10.7 \%$ & $32.6 \%$ & $9.1 \%$ \\
\hline PTEN & $35.2 \%$ & $0.0 \%$ & $29.3 \%$ & $2.0 \%$ \\
\hline
\end{tabular}


Table 1 continued

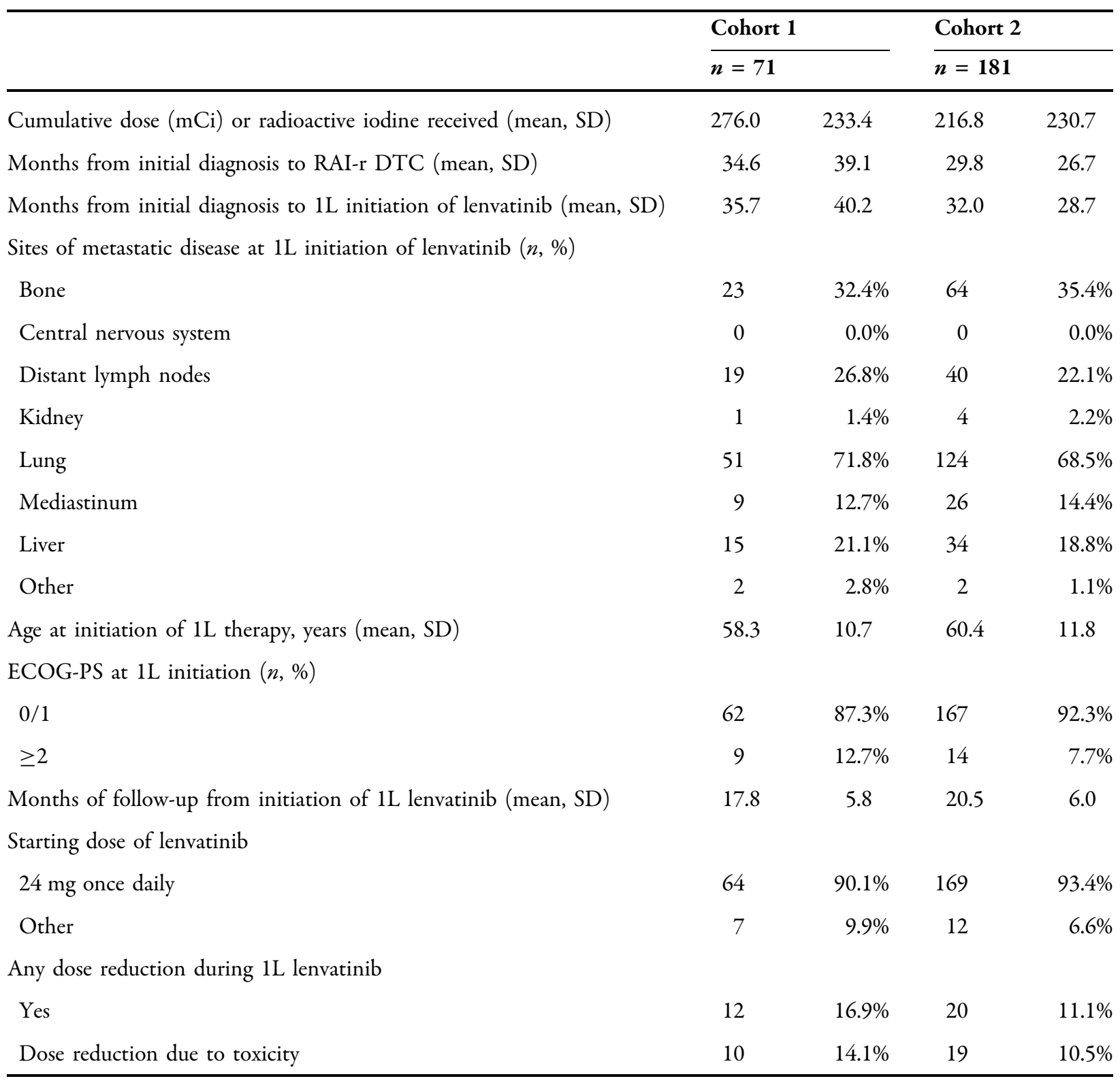

$D T C$ differentiated thyroid cancer, $I L$ first-line, $S D$ standard deviation, ECOG-PS Eastern Cooperative Oncology Group performance status

The most common 2L treatment regimen following $1 \mathrm{~L}$ lenvatinib was sorafenib $(49.7 \%)$ followed by cabozantinib (19.3\%). Additional therapies received in $2 \mathrm{~L}(n=56)$ in order of frequency were pazopanib $(n=15)$, sunitinib $(n=10)$, vandetinib $(n=8)$, paclitaxel $(n=7)$, axitinib $(n=6)$, dabrafenib/trametinib combination $(n=5)$, and pembrolizumab $(n=2)$. The mean follow-up from initiation of $2 \mathrm{~L}$ was
7.1 months ( $\mathrm{SD}=5.3$ ). The ORR to $2 \mathrm{~L}$ therapy was $15.5 \%$ (Table 4 ) and by treatment regimen, 2L ORRs were $11.1 \%$ for sorafenib, $14.3 \%$ for cabozantinib, and $23.2 \%$ for other $2 \mathrm{~L}$ therapies.

At the time of data cutoff, $62.4 \%$ of patients $(n=113)$ in cohort 2 were still receiving $2 \mathrm{~L}$ therapy, $6.6 \%(n=12)$ were receiving third-line treatment, $28.7 \%(n=52)$ were deceased, $1.1 \%$ $(n=2)$ were receiving only palliative care or had 
Table 2 Real-world ORR with 1L lenvatinib

\begin{tabular}{|c|c|c|c|c|}
\hline & Co & & & \\
\hline & $\overline{n=}$ & & & \\
\hline & $N$ & $\%$ & $n$ & $\%$ \\
\hline $\mathrm{ORR}^{\dagger}(\%)$ & 46 & 64.8 & 97 & 53.6 \\
\hline $\mathrm{CR}$ & 6 & 8.5 & 3 & 1.7 \\
\hline PR & 40 & 56.3 & 94 & 51.9 \\
\hline SD & 10 & 14.1 & 45 & 24.9 \\
\hline PD & 10 & 14.1 & 26 & 14.4 \\
\hline Not evaluable & 5 & 7.0 & 13 & 7.2 \\
\hline
\end{tabular}

$I L$ first-line, $C R$ complete response, $O R R$ overall response rate, $P D$ progressive disease, $P R$ partial response, $S D$ stable disease

$\dagger$ Per RECIST v1.1 criteria; see "Methods"

been admitted to hospice, and $1.1 \%(n=2)$ had been lost of follow-up. Median PFS to 2L treatment overall (Table 3) was 10.5 months (95\% CI 7.3-not reached). Median PFS by treatment regimen in 2L (Fig. 2) was 10.8 months (95\% CI 5.4-not reached) for patients treated with sorafenib, not reached for patients treated with cabozantinib, and 10.5 months (95\% CI 5.5-not reached) for patients treated with other therapies in 2L.

At data cutoff, 52 patients were deceased: $34 / 52$ deaths were recorded for patients treated with $2 \mathrm{~L}$ sorafenib, 1/52 in cabozantinib-treated patients, and $17 / 52$ in patients who received other $2 \mathrm{~L}$ therapies. The OS from initiation of $1 \mathrm{~L}$ lenvatinib in cohort 2 was $92.8 \%(95 \%$ CI 89.0-96.6\%), 81.5\% (95\% CI 75.7-87.2\%), and $66.9 \%(95 \%$ CI $58.8-75.0 \%)$ at 12,18 and 24 months, respectively.

\section{DISCUSSION}

This is the first and largest real-world study in the USA to evaluate outcomes of 1L lenvatinib therapy and post-lenvatinib therapy for patients with RAI-r DTC. After approval in 2015, lenvatinib replaced sorafenib as the most commonly prescribed 1L treatment for DTC, representing $43.4 \%$ and $66.7 \%$ of $1 \mathrm{~L}$ treatment in 2015 and 2016, respectively [14]. These real-world data provide supporting evidence that when prescribed in the community setting, up to $64.8 \%$ of patients treated with lenvatinib in 1L experienced disease response (similar to that observed in the pivotal SELECT trial) [13]. In cohort 2 in which all patients received $2 \mathrm{~L}$ agents post 1L lenvatinib, the median PFS was 14.0 months, disease response in $2 \mathrm{~L}$ across diverse therapies was observed in $15.5 \%$ of patients, and the 2L median PFS following $1 \mathrm{~L}$ lenvatinib was 10.5 months. Dose reductions due to toxicity were infrequent, occurring in a maximum of $16.9 \%$ of patients. The major difference in the cohort selection criteria between SELECT and this study was that SELECT allowed patients to receive one prior tyrosine kinase inhibitor (TKI) and hence included treatmentnaïve plus pre-treated patients with DTC while

Table 3 PFS from initiation of 1L lenvatinib and 2L post-lenvatinib (cohort 2)

\begin{tabular}{lll}
\hline & $\begin{array}{l}\text { 1L lenvatinib } \\
\text { Estimate }(\mathbf{9 5 \%} \mathbf{C I})\end{array}$ & $\begin{array}{l}\text { 2L post-lenvatinib } \\
\text { Estimate (95\% CI) }\end{array}$ \\
\hline Median months & $14.0(12.7-15.0)$ & $10.5(7.3$-not reached) \\
6-month & $86.0 \%(80.9-91.1 \%)$ & $63.0 \%(54.5-71.6 \%)$ \\
12-month & $60.3 \%(53.0-67.6 \%)$ & $47.7 \%(36.6-58.8 \%)$ \\
18-month & $21.2 \%(14.8-27.5 \%)$ & $\dagger$ \\
24-month & $2.1 \%(0.0-4.5 \%)$ & $\dagger$ \\
\hline
\end{tabular}

$I L$ first-line, $2 L$ second-line, $C I$ confidence interval, $P F S$ progression-free survival

$\dagger$ No events (progression or death) were observed after 12 months 
Table 4 Real-world ORR with 2L therapies post discontinuation of 1L lenvatinib

\begin{tabular}{|c|c|c|c|c|c|c|c|c|}
\hline & \multirow{2}{*}{\multicolumn{2}{|c|}{$\begin{array}{l}\text { Across all } 2 \mathrm{~L} \text { therapies } \\
n=181\end{array}$}} & \multirow{2}{*}{\multicolumn{2}{|c|}{$\begin{array}{l}\text { Sorafenib } \\
n=90\end{array}$}} & \multirow{2}{*}{\multicolumn{2}{|c|}{$\begin{array}{l}\text { Cabozantinib } \\
n=35\end{array}$}} & \multirow{2}{*}{\multicolumn{2}{|c|}{$\begin{array}{l}{\text { Other } 2 \mathrm{~L} \text { therapies }^{\dagger}}_{n=56}\end{array}$}} \\
\hline & & & & & & & & \\
\hline & $\bar{n}$ & $\%$ & $\bar{n}$ & $\%$ & $\bar{n}$ & $\%$ & $\bar{n}$ & $\%$ \\
\hline ORR (\%) & 28 & 15.5 & 10 & 11.1 & 5 & 14.3 & 13 & 23.2 \\
\hline CR & 0 & 0.0 & 0 & 0.0 & 0 & 0.0 & 0 & 0.0 \\
\hline PR & 28 & 15.5 & 10 & 11.1 & 5 & 14.3 & 13 & 23.2 \\
\hline SD & 54 & 29.8 & 25 & 27.8 & 11 & 31.4 & 18 & 32.1 \\
\hline PD & 29 & 16.0 & 19 & 21.1 & 1 & 2.9 & 9 & 16.1 \\
\hline Not evaluable* & 70 & 38.7 & 36 & 40.0 & 18 & 51.4 & 16 & 28.6 \\
\hline
\end{tabular}

$I L$ first-line, $2 L$ second-line, $C R$ complete response, $O R R$ overall response rate, $P D$ progressive disease, $P R$ partial response, $S D$ stable disease

${ }^{*}$ Not evaluable includes patients for whom provider reported best response to therapy had not yet occurred/radiographic imaging to confirm response had not yet been conducted

$\dagger$ Include pazopanib $(n=15)$, sunitinib $(n=10)$, vandetinib $(n=8)$, paclitaxel $(n=7)$, axitinib $(n=6)$, dabrafenib/trametinib combination $(n=5)$, and pembrolizumab $(n=2)$

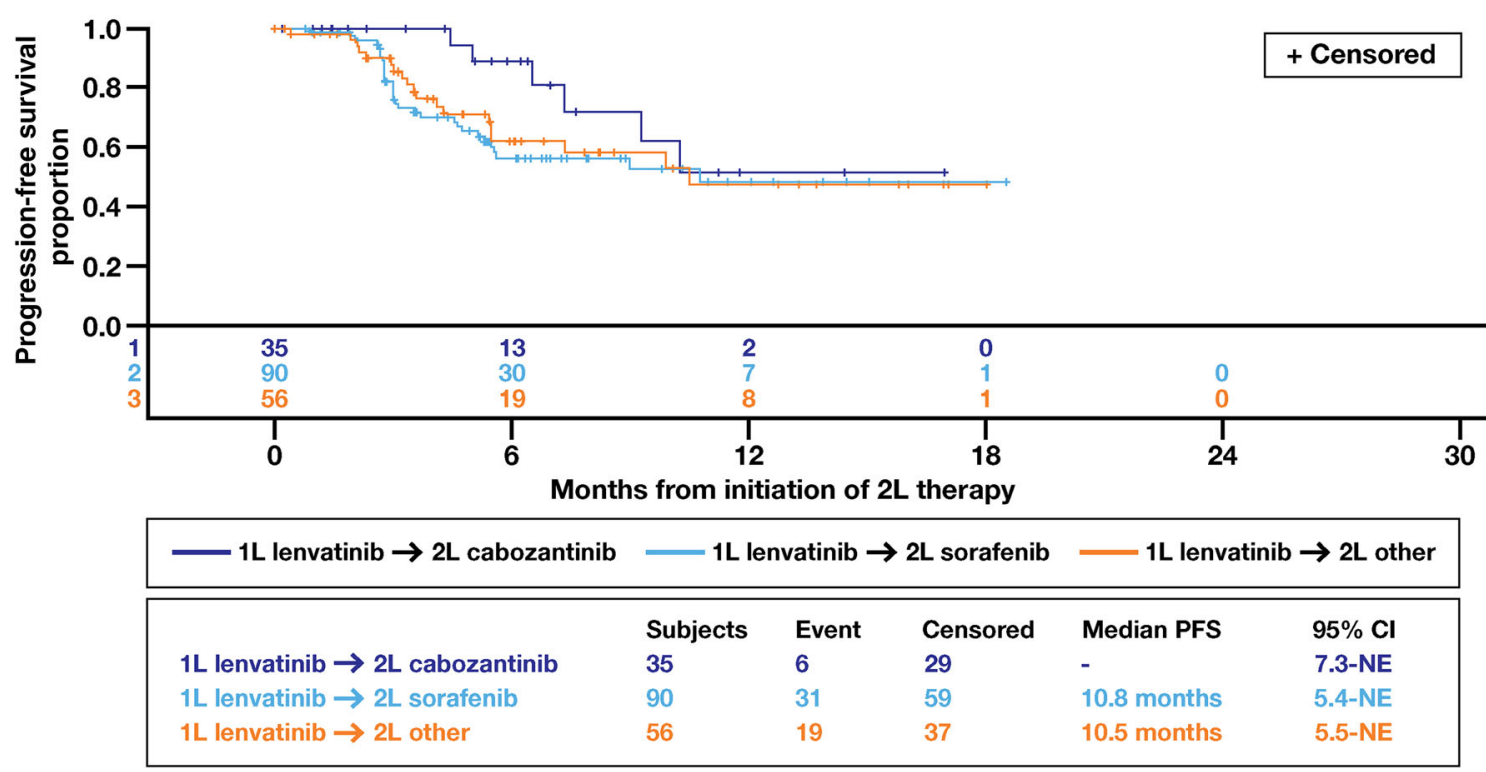

Fig. 2 Kaplan-Meier curve for PFS of $2 \mathrm{~L}$ therapy post lenvatinib by $2 \mathrm{~L}$ treatment regimen. $1 \mathrm{~L}$ first-line, $2 \mathrm{~L}$ second-line, $\mathrm{CL}$ confidence limit, NE not evaluable

the current study was focused only on patients who received $1 \mathrm{~L}$ lenvatinib (i.e., treatmentnaïve patients). Other differences in the selection criteria apply as a result of the nature of retrospective studies, such as ECOG-PS 0-2, which independently reviewed radiologic evidence of progression within the previous 13 months. Several studies have evaluated the real-world clinical outcomes of lenvatinib for treating patients with RAI-r DTC in Europe 
[16-19]. Most of these studies concluded that there was a real-world clinical benefit of using lenvatinib to treat patients with RAI-r DTC in four European countries. However, the sample size for these studies was smaller, ranging from 12 to 75 patients.

Our study consisted of two types of patients treated with lenvatinib-those who had received $1 \mathrm{~L}$ therapy and were still receiving lenvatinib at the time of data cutoff and those who had discontinued 1L lenvatinib therapy and initiated a $2 \mathrm{~L}$ treatment. For those patients still receiving lenvatinib at the time of data cutoff (cohort 1), patients remained on therapy for 17.8 months and the ORR estimate was similar to that observed in the SELECT phase III trial [13]. Among the cohort of patients who had discontinued $1 \mathrm{~L}$ lenvatinib during the study period and initiated a 2L therapy (cohort 2), the ORR was $53.6 \%$ and the median PFS was 14.0 months. These real-world estimates in cohort 2 are lower when indirectly compared to the SELECT trial. We note that the selection criteria for cohort 2 (having to have failed 1L lenvatinib by data cutoff) may result in patients with a more aggressive course of disease being selected for study inclusion. Of note, patients in cohort 2 had a much higher frequency of genetic abnormalities compared to patients in cohort 1.

In cohort 2, nearly half of the patients $(49.7 \%)$ received sorafenib as $2 \mathrm{~L}$ therapy; and the ORR for $2 \mathrm{~L}$ sorafenib was $11.1 \%$ and the median PFS was 10.8 months. In comparison, the ORR and median PFS in the DECISION trial (sorafenib versus placebo in treatment-naïve patients) were $12.2 \%$ and 10.8 months, respectively, similar to that observed in this real-world cohort of patients receiving sorafenib in 2L [20]. For cabozantinib, prescribed in $19.3 \%$ of patients in our study, the ORR was $14.3 \%$ and the median PFS was not reached.

\section{Limitations}

As a retrospective observational study of secondary data from multiple sites, biases in provider participation, patient selection, and information may exist and impact our findings.
We could not require that providers submit all patients treated by them or at their site; however, given the rarity of RAI-r DTC, the specificity of our selection criteria, and limiting providers to submitting a maximum of 10 cases, we believe that this bias is minimal. Second, radiology reports and images were not evaluated by an independent reviewer and inaccurate recording of tumor lesions by the patient's treating provider may have occurred. Finally, our design inherently may create downwardly biased estimates of 1L PFS (requirement of failure of $1 \mathrm{~L}$ therapy within the study period) and upwardly biased estimates of PFS for 2L therapy following 1L lenvatinib (high rate of early censoring). In addition, although it is not included in this current study given the small sample size for most $2 \mathrm{~L}$ agents, more data is warranted to assess the impact of patients' characteristics on the selection of $2 \mathrm{~L}$ treatments.

\section{CONCLUSIONS}

In this real-world examination of patients with RAI-r DTC, the results demonstrate that patients who were prescribed $1 \mathrm{~L}$ treatment with lenvatinib achieved real-world disease response rates consistent with those observed in the pivotal clinical trials. Moreover, for those patients who received a $2 \mathrm{~L}$ therapy after $1 \mathrm{~L}$ lenvatinib, clinical benefit across multiple therapies was observed, thus providing several potential $2 \mathrm{~L}$ options for these patients. Further comparative effectiveness research is warranted to understand the long-term outcomes of various treatment sequences to aid clinicians and patients with identifying an appropriate treatment strategy.

\section{ACKNOWLEDGEMENTS}

The authors would also like to thank Edward Wang, PharmD, MBA, Russel Knoth, MA, PhD, (formerly of Eisai Inc.) and Callan Myerscough, BA (formerly of Cardinal Health) for their contributions to study design. 
Funding. This study and the journal's Rapid Service and Open Access Fees were funded by Eisai Inc., Woodcliff Lake, NJ, USA, and Merck Sharp \& Dohme Corp., a subsidiary of Merck \& Co., Inc., Kenilworth, NJ, USA.

Authorship. All named authors meet the International Committee of Medical Journal Editors (ICMJE) criteria for authorship for this article, take responsibility for the integrity of the work as a whole, and have given their approval for this version to be published.

Disclosures. JKK, HY, DL, and BAF are employees of Cardinal Health who received funding to design and conduct the research. YW is an employee of Eisai Inc. DC was an employee of Eisai Inc. at the time of research was conducted, and currently an employee of Boehringer Ingelheim.

Compliance with Ethics Guidelines. Prior to data collection, the research protocol and eCRF were reviewed by the Western IRB (Study Number: 1187046). The IRB found that this research meets the requirements for a waiver of consent under 45 CFR 46.116(d).

Data Availability. Datasets generated during and/or analyzed during the current study will not be deposited.

Open Access. This article is licensed under a Creative Commons Attribution-NonCommercial 4.0 International License, which permits any non-commercial use, sharing, adaptation, distribution and reproduction in any medium or format, as long as you give appropriate credit to the original author(s) and the source, provide a link to the Creative Commons licence, and indicate if changes were made. The images or other third party material in this article are included in the article's Creative Commons licence, unless indicated otherwise in a credit line to the material. If material is not included in the article's Creative Commons licence and your intended use is not permitted by statutory regulation or exceeds the permitted use, you will need to obtain permission directly from the copyright holder. To view a copy of this licence, visit http://creativecommons.org/licenses/bync/4.0/.

\section{REFERENCES}

1. Surveillance, Epidemiology and End Results (SEER) Cancer Stat Facts: Thyroid Cancer. https://seer. cancer.gov/statfacts/html/thyro.html. Accessed 15 Aug 2019.

2. Cooper DS, Doherty GM, Haugen BR, et al. Revised American Thyroid Association management guidelines for patients with thyroid nodules and differentiated thyroid cancer: the American Thyroid Association (ATA) Guidelines Taskforce on Thyroid Nodules and Differentiated Thyroid Cancer. Thyroid. 2009;19(11):1167-214.

3. Hundahl SA, Fleming ID, Fremgen AM, Menck HR. A national cancer data base report on 53,856 cases of thyroid carcinoma treated in the U.S., 1985-1995. Cancer. 1998;19(11):1167-214.

4. Mazzaferri EL, Jhiang SM. Long-term impact of initial surgical and medical therapy on papillary and follicular thyroid cancer. Am J Med. 1994;97(5):418-28.

5. Samaan NA, Schultz PN, Haynie TP, Ordonez NG. Pulmonary metastasis of differentiated thyroid carcinoma: treatment results of 101 patients. J Clin Endocrinol Metab. 1985;60(2):376-80.

6. Ruegemer JJ, Hay ID, Bergstralh EJ, Ryan JJ, Offord $\mathrm{KP}$, Gorman CA. Distant metastases in differentiated thyroid carcinoma: a multivariate analysis of prognostic variables. J Clin Endocrinol Metab. 1988;67(3):501-8.

7. Durante C, Haddy N, Baudin E, et al. Long-term outcome of 444 patients with distant metastases from papillary and follicular thyroid carcinoma: benefits and limits of radioiodine therapy. J Clin Endocrinol Metab. 2006;91(8):2892-9.

8. Brown RL, de Souza JA, Cohen EE. Thyroid cancer: burden of illness and management of disease. J Cancer. 2011;2:193-9.

9. Xing M, Haugen BR, Schlumberger M. Progress in molecular-based management of differentiated thyroid cancer. Lancet. 2013;381(9871):1058-69.

10. Schmidt A, Iglesias L, Klain M, Pitoia F, Schlumberger MJ. Radioactive iodine-refractory differentiated thyroid cancer: an uncommon but challenging situation. Arch Endocrinol Metab. 2017;61(1):81-9. 
11. Jayarangaiah A, et al. Therapeutic options for advanced thyroid cancer. Int J Clin Endocrinol Metab. 2019;5(1):26-34.

12. LENVIMA $^{\circledR}$ (lenvatinib). Full Prescribing Information. Eisai Inc, Woodcliff Lake, 2018.

13. Schlumberger $M$, et al. Lenvatinib versus placebo in radioiodine-refractory thyroid cancer. N Engl J Med. 2015;372(7):621-30.

14. Dacosta Byfield SA, et al. Real-world treatment patterns among patients initiating small molecule kinase inhibitor therapies for thyroid cancer in the United States. Adv Ther. 2019;36(4):896-915.

15. Eisenhauer EA, Therasse P, Bogaerts J, et al. New response evaluation criteria in solid tumours: revised RECIST guideline (version 1.1). Eur J Cancer. $2009 ; 45(2): 228-47$.

16. Aydemirli MD, et al. Effectiveness and toxicity of lenvatinib in refractory thyroid cancer: Dutch reallife data. Eur J Endorcinol. 2020;182(2):131-8.
17. Balmelli $\mathrm{C}$, et al. Lenvatinib in advanced radioiodine-refractory thyroid cancer-a retrospective analysis of the swiss lenvatinib named patient program. J Cancer. 2018;9(2):250-5.

18. Berdelou A, et al. Lenvatinib for the treatment of radioiodine-refractory thyroid cancer in real-life practice. Thyroid. 2017. https://doi.org/10.1089/ thy.2017.0205.

19. Nervo A, et al. Lenvatinib in advanced radioiodinerefractory thyroid cancer: a snapshot of real-life clinical practice. Anticancer Res. 2018;38(3): 1643-9.

20. Brose MS, Nutting CM, Jarzab B, et al. Sorafenib in radioactive iodine-refractory, locally advanced or metastatic differentiated thyroid cancer: a randomized, double-blind, phase 3 trial. Lancet. 2014;384(9940):319-28. 